\title{
The role of ultrasound imaging at detecting aortic aneurysm in emergency department
}

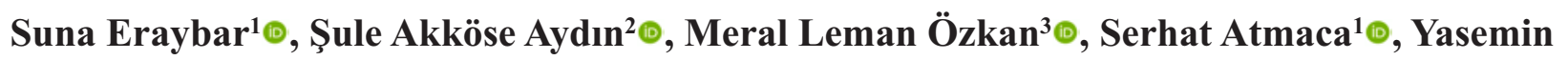 \\ Nennicioğlu ${ }^{1} \odot$ \\ ${ }^{I}$ Department of Emergency Medicine, University of Health Sciences, Bursa Yüksek Ihtisas Training and Research Hospital, Bursa, \\ Turkey \\ ${ }^{2}$ Department of Emergency Medicine, Uludă̆ University of School of Medicine, Bursa, Turkey \\ ${ }^{3}$ Department of Emergency Medicine, Çekirge State Hospital, Bursa, Turkey
}

DOI: $10.18621 /$ eurj.402076

\begin{abstract}
Objective: Our study was carried out to investigate the effects of focused ultrasound imaging which is performed by emergency physicians in diagnosis and duration of treatment of abdominal aortic aneurysm.

Methods: The patients over 50 years; who applied to Uludağ University Emergency Department with the complaints of abdominal pain, side pain, chest pain, syncope, unexplained hypotension and under suspicion of the abdominal aortic aneurysm, were included in the study. Bedside ultrasound for abdominal aorta was done and diameter measurements of aorta recorded in order to determine the presence of an abdominal aortic aneurysm or to exclude the diagnosis.

Results: A total of 133 patients were included in the study. Eight patients were excluded from the study because of inadequate bedside US imaging. The aortic diameter was measured as $\geq 3 \mathrm{~cm}$ in the $54(43.2 \%)$ patients. The aortic diameter was found more than $5 \mathrm{~cm}$ in $8(6.4 \%)$ patients. After ultrasonographic investigations, aneurysm rupture $(\mathrm{n}=5 ; 4 \%)$, aortic dissction $(\mathrm{n}=13 ; 10.4 \%)$ and aortic aneurysm $(\mathrm{n}=36 ; 28.8 \%)$ were detected. The diagnosis was confirmed with computed tomography in all patients who had aortic pathology. Because of the continuance of clinic suspects in the patients whose aortic diameters were less than $3 \mathrm{~cm}$, their computed tomography images were obtained and their aortic diameters were found within normal limits. Further imaging studies weren't performed at the remaining 48 (38.4\%) patients because different prediagnosis was considered. Emergency ultrasound had a sensitivity of $100 \%$ (95\% CI: 87-100), a specificity of $91 \%$ (95\% CI: 90.8-99.8).

Conclusions: The diagnosis of abdominal aortic aneurysms is omitted $30 \%$ in emergency departments. It has a high morbidity and mortality if the diagnosis is skipped. In the presence of aortic aneurysm suspicion, evaluation of aortic diameter by bedside ultrasound is diagnostic. Bedside ultrasound evaluation by emergency physicians should become routine for using time and investigation numbers properly.
\end{abstract}

Keywords: emergency department, abdominal aortic aneurysms, bedside ultrasound

Received: March 5, 2018; Accepted: May 2, 2018; Published Online: August 29, 2018

cute abdominal pain is a symptom which is presently less than a week, caused by non-traumatic reasons, developed in the progress of patholo- gies of abdominal or non-abdominal organs. Acute abdominal pain is the most important symptom of surgical or medical emergencies [1]. Abdominal pain

Address for correspondence: Suna Eraybar, MD., University of Health Sciences, Bursa Yüksek Ihtisas Training and Research Hospital, Department of Emergency Medicine, Mimar Sinan Mah., Emniyet Cad., Yıldırım, Bursa, Turkey

E-mail: sunaeraybar@gmail.com 
constitutes $5-10 \%$ of all emergency care applications [2]. Diagnosis and treatment of acute abdominal pain at emergency departments; is still one of the important clinical problems despite all the technological advancement. Especially at the situations when the patient is not stable, there are some problems at using imaging methods which origin from transporting difficulties.

Aortic aneurysms are at the $13^{\text {th }}$ rank of most common death reasons in the USA [3]. Appraisal rate is $10 / 100.000$ per year and it's estimated higher in elder groups. The frequency of acute aortic dissection is approximately 5.2 in a million [4] and detected as $1.5 \%$ at $60-80$ age group in Turkey [5]. The frequency of aortic dissection is 2 to 5 times more in men than women. The diameter of an aneurysm is an important factor at detecting the rupture risk [6]. At many studies, it is shown that smoking affects the enlargement of an aneurysm [7-10]. It is detected that chronic obstructive lung disease increases the risk of aortic aneurysm rupture approximately 3.6 times [11].

Most of the patients who applied with acute aortic dissection describe a chest pain in a tearing character. The character of pain and radiating from chest to back and sometimes to waist is a very important clinical sign $[10,12]$. If aortic dissections are left to their own natural course, they become substantially life threating. So, it must be diagnosed quickly and life-saving interventions must be started urgently. At this point, usage of diagnostic imaging methods at the emergency department has vital importance.

Ultrasound (US) is a conventional imaging method at differentiating between aneurysmatic and normal aorta. It has many important advantages like; no exposure to ionising radiation during imaging or any usage of contrast substances and it can be performed at the bedside [14]. The aim of the our study was investigate the effects of focused ultrasound imaging which is performed by emergency physicians in diagnosis and duration of treatment of abdominal aortic aneurysm (AAA) suspicion.

\section{METHODS}

Our study was performed prospectively for one year period at the Emergency Department of Uludağ University School of Medicine. Institutional ethics committee approval was taken for our study.

The patients over 50 years old; who admitted to the emergency department with the complaints of abdominal pain, side pain, chest pain, syncope, unexplained hypotension and who are under suspicion for an aortic aneurysm, were included to study. Diameter measurement and abdominal aorta imaging were performed by bedside ultrasound for detecting or excluding the abdominal aortic aneurysm in these patients.

Patients age, application clinics, vital signs, smoking history, co-morbidities, the presence of the abdominal aortic aneurysm in family history, known abdominal aortic aneurysm history, undergone aortic and other cardiac surgeries were recorded.

Informations like advanced age; hypertension history; severe abdominal, back and side pain in tearing character, syncope after abdominal pain, unexplained hypotension and also some physical examination signs like pulsatile mass at abdomen, tension arterial difference between right and left arm, difference at peripheral pulses, presence of focal neurologic deficit were noted as a value in suspicion of AAA.

Abdominal aortic diameter measurement was performed by bedside ultrasound imaging after stabilization of patients. Measurements were performed by emergency physicians who finished training course which is proper to US usage at emergency department protocol of American College of Emergency Physicians and that is arranged by Emergency Medicine Physicians Association of Turkey. US measurements were performed with Siemens Digital Color Doppler Ultrasound SIUI Apogee 3500 device, measurements were done at mode B with $3.5 \mathrm{MHz}$ convex probe.

Abdominal aorta measurement was performed at the grey window, from diaphragmatic hiatus to aortic bifurcation on the transverse and longitudinal planes. Wall-to-wall diameter measurements from the widest area of aorta were recorded as $<3 \mathrm{~cm}, 3-5 \mathrm{~cm}$, and $>$ $5 \mathrm{~cm}$. Measurements which are $\geq 3 \mathrm{~cm}$ were accepted as aneurysmatic dilatation. Aneurysm area was defined as suprarenal or infrarenal according to renal artery exit point. When the aneurysmatic segment was detected; its progress, presence of flap and intraaortic thrombus were recorded. Hepatorenal, splenorenal and pelvic regions were investigated ultrasonographically 
for abdominal free fluid after the aortic measurement.

Contrast-enhanced thoracic and abdominopelvic computerized tomography (CT) imaging was performed for all of the patients whose measurements were $\geq 3 \mathrm{~cm}$. Information about patients' clinic was also given synchronously to the cardiovascular surgery clinic.

In the presence of ongoing clinical suspicion we applied further imaging methods despite aortic diameter has measured $<3 \mathrm{~cm}$. For final diagnosis computed tomography were accepted as the golden standard for showing ultrasonography's sensitivity. CT was used at the situations where aortic diameter measurements couldn't be evaluated clearly or completely at the suprarenal or infrarenal levels. These patients were excluded from the study.

\section{Statistical Analysis}

All data belong to study were recorded via SPSS 13.0 for windows. Standard deviation $( \pm)$ at continuous variables and $\mathrm{n}$ and \% values at categorical variables, were used as descriptive statistics. Pearson chisquared test, Fisher's exact chi-square test, and Mcnemar's test were used at comparing the categorical variables. $P<0.05$ was accepted as statistically significant. Sensitivity and specificity values, positive and negative predictive values were calculated for evaluating the effectiveness of Computed Tomography and US at diagnosing.

\section{RESULTS}

Our study included 133 patients over the age of 50. Eight patients were excluded from the study because of inadequate bedside US imaging due to dense abdominal gas $(n=3)$, diffuse defence $(n=2)$, and obesity $(n=3)$. Computed tomography was used for excluding AAA in these patients. As a result, 125 patients who had bedside US imaging for the suspect of AAA at emergency room included to the study.

According to our study population 78 (62.4\%) were male and remaining $47(37.6 \%)$ were female. Mean age of patients was 67 yeras (min: 50 - max:90, std deviation 9.857). The most common complaint was an abdominal pain with $84(67.2 \%)$ patients. Patients who had more than one complaint, symptoms were recorded separately. Most common complaints are summarized in Table 1. Smoking history was present in the $51.2 \%(n=64)$ of the study population. Physical examination findings which support AAA; were evaluated as pulsatile mass at the abdomen, difference at peripheral pulses, tension arterial difference between right and left arm. There is a pulsatile mass in $18(14.4 \%)$ patients, tension arterial difference between the arms in $24(19.2 \%)$ patients, the difference at peripheral pulses in $21(16.8 \%)$ patients.

After the bedside US evaluation, the aortic diameter was measured $\geq 3 \mathrm{~cm}$ in $54(43.2 \%)$ patients. The aortic diameter was $>5 \mathrm{~cm}$ in $8(6.4 \%)$ patients. False lumen, flap, intraaortic thrombus and abdominal free fluid were investigated with US in all the patients who had a $3 \mathrm{~cm}$ aortic diameter. Flap in 14 (11.2\%) patients, intraaortic thrombus in $27(21,6 \%)$ patients, abdominal free fluid in 5 (4\%) patients were detected. As a result of US investigations; AAA rupture in 5 (4\%) patients, aortic dissection in $13(10.4 \%)$ patients, and aortic aneurysm signs in $36(28.8 \%)$ patients were detected. IV contrast-enhanced thoracic and abdominopelvic tomography imaging was planned for these patients. Clear results were acquired from 53 patients except for one patient, who was sent for computed tomography imaging after emergency evaluation and his situation was worsened during iv contrast enhancement. Aneurysmatic dilatation and flap presence with the false lumen at aorta was reported but dissection typology couldn't be performed due to unfinished sequencing and the report was interpreted inadequate evaluation.

Further imaging was performed due to the presence of ongoing clinical suspect by their physicians in $23(18.4 \%)$ patients although their aortic

Table 1. The presenting symptoms of patients*

\begin{tabular}{lcc}
\hline Symptom & Frequency (n) & Percent (\%) \\
\hline Abdominal pain & 84 & 67.2 \\
Back pain & 61 & 48.8 \\
Flank pain & 34 & 27.2 \\
Chest pain & 15 & 12.0 \\
Syncope & 17 & 13.6 \\
Hypotension & 3 & 2.4 \\
Neurological deficits & 10 & 8 \\
Cardiopulmonary arrest & 1 & 0.8 \\
\hline
\end{tabular}

*Some patients had more than one symptom. 
diameters were measured as $<3 \mathrm{~cm}$ at US investigations. Aortic calibrations of these patients detected between normal range at $\mathrm{CT}$ and there were no signs of AAA or its complications. Thorax and abdominopelvic $\mathrm{CT}$ imaging were performed in total 77 patients.

Dissection typology of patients who got aortic dissection diagnosis by CT was performed by DeBakey classification. Types were recorded except one patient whose situation became worsen and CT imaging couldn't be finished. According to this, type 1 aortic dissection in $7(53.8 \%)$ patients and type 3 aortic dissection in $5(38.4 \%)$ patients were detected. At the remaining 48 (38.4\%) patients despite other prediagnoses, aortic diameter measurement was performed with US for excluding AAA. The aortic diameter was measured as $<3 \mathrm{~cm}$ in these patients and further imaging wasn't performed. Additional imaging methods were used which belong their prediagnoses. Flowchart of diagnostic approaches of patients after bedside aortic US imaging, are shown at Figure 1.

In our study $29(23.2 \%)$ patients have aortic aneurysm diagnosis whereas, fatal aortic pathologies which include aortic dissection and aneurysm rupture were detected in $18(14.4 \%)$ patients. Nephrolithiasis was detected as the most common secondary diagnosis which clinically interferes with AAA at the patients whose aortic diameters were measured with US. Patients' final diagnoses are defined in Table 2.

Distribution of sex, co-morbidities, smoking history, physical examination signs of 29 patients who had AAA diagnosis and 18 patients who had aneurysm rupture or dissection, were evaluated retrospectively. Thirty-six (76.6\%) of 47 patients were male and the remaining $11(23.4 \%)$ were female. Thirty-nine $(82.39 \%)$ patients were following for hypertension and there was additional medication usage. Male sex and hypertension effect at AAA diagnosis was determined as statistically significant $(p=0.01)$.

When the relation is calculated between the final diagnoses and smoking history, 70.2\% $(n=33)$ of them has smoking history and smoking history was determined as an independent risk factor at AAA diagnosis $(p<0.03)$.

Aneurysm presence was considered in 54 patients after abdominal aortic evaluation with US at the emergency room but the aneurysmatic segment wasn't seen after $\mathrm{CT}$ imaging. In the light of these false positive ratios, the sensitivity of US at the diagnosis of AAA was calculated as 100\% (95\% CI: 87-100) and specificity of it calculated as $91 \%$. Diagnosis is propounded by all the patients whose aortic diameters were measured $>3 \mathrm{~cm}$ with US. At the patients, whose US investigations didn't show any an aneurysm but still got under CT imaging (7/125), any additional benefit of undergone imaging wasn't detected for aortic pathology. Positive predictive value of US is determined as $87 \%$, and negative predictive value is detected as $100 \%$. Sixty-one $(48.8 \%)$ patients were hospitalized because of their diagnoses. Twenty-six

Table 2. The distribution of final diagnoses

\begin{tabular}{lcc}
\hline Final diagnoses & Frequency (n) & Percent (\%) \\
\hline AAA & 29 & 23.2 \\
Aortic dissection & 13 & 10.4 \\
Aneurysm rupture & 5 & 4.0 \\
Other & & \\
$\quad$ Nephrolithiasis & 23 & 18.4 \\
Acute coronary syndrome & 14 & 11.2 \\
Cholelithiasis & 12 & 9.6 \\
Acute pancreatitis & 10 & 8.0 \\
Activation of peptic ulcus & 9 & 7.2 \\
Arterial occlusion & 4 & 3.2 \\
Pulmonary embolism & 2 & 1.6 \\
Cerebrovascular event & 2 & 1.6 \\
Mesenteric iscemia & 1 & 0.8 \\
Renal infarction & 1 & 0.8 \\
Total & 125 & 100 \\
\hline
\end{tabular}

AAA = Abdominal aortic aneurysm 

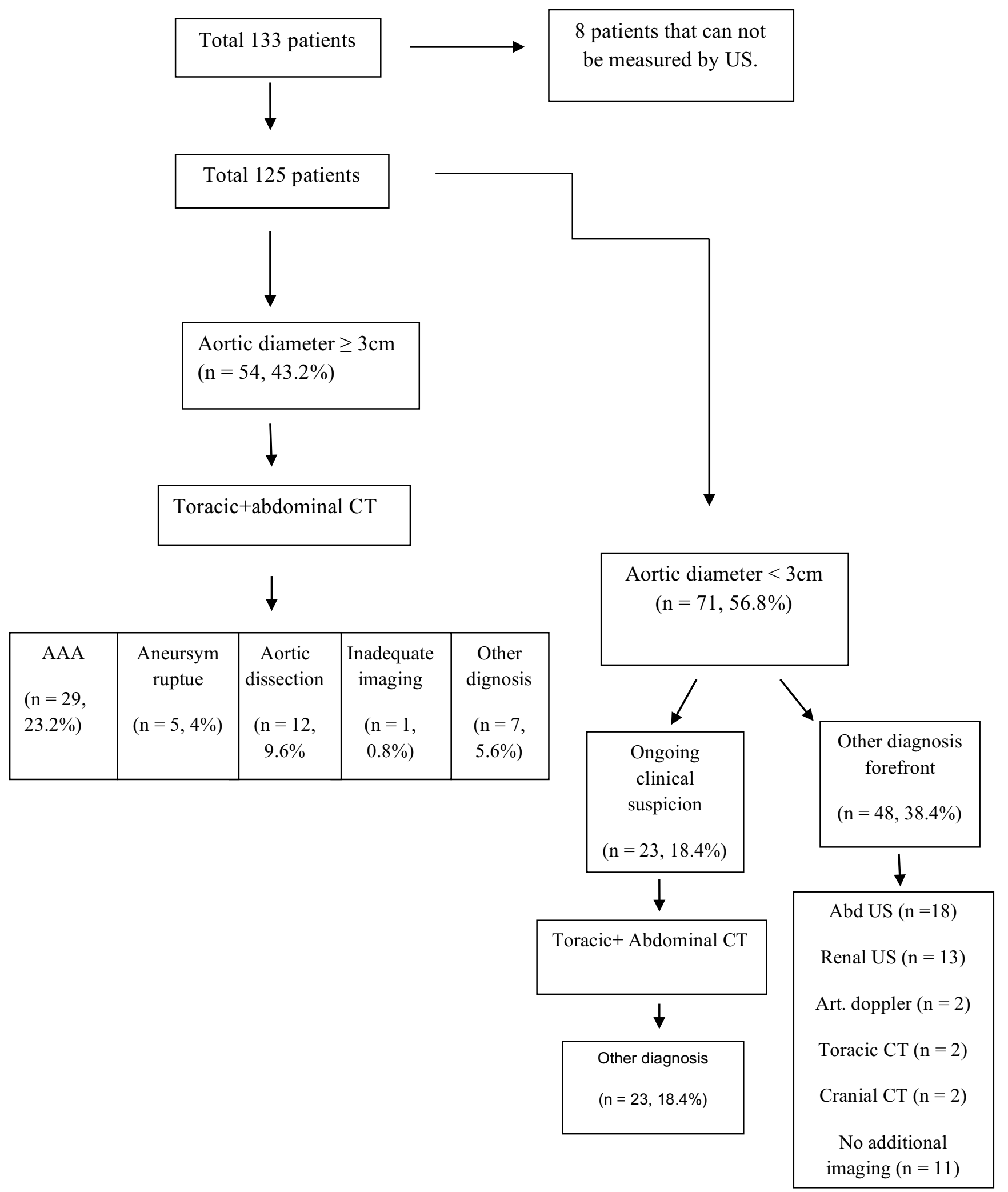

Figure 1: The distribution of additional imaging after emergency US. AAA =Abdominal Aortic Aneurysm, US = Ultrasound, CT = Computerized tomography, Abd = Abdominal. 
(20.8\%) patients were admitted to cardiovascular surgery intensive care unit and $14(11.2 \%)$ patiens were admitted to coronary intensive care unit. One patient who got mesenteric ischemia diagnosis and total $7(5.6 \%)$ patients who had acute cholecystitis and acute pancreatitis diagnoses were admitted to general surgery service.

One patient who admitted to emergency room with hypotension and abdominal pain, and bedside US aortic diameter measurement was $>3 \mathrm{~cm}$, also flap and intraaortic thrombus in US imaging detected were taken to $\mathrm{CT}$ imaging with the prediagnosis of aortic dissection but during imaging, arrest developed. There was another patient, who applied with the complaints of syncope and right leg pain and right leg femoral artery and distal couldn't be palpated; aortic diameter was measured as $<3 \mathrm{~cm}$ but the clinical exclusion of diagnosis couldn't be done so CT imaging was performed. Thrombus was detected in the iliac artery. Both of these patients were resuscitated at the emergency room but they didn't respond and they're accepted as exitus.

Five (4\%) patients left the emergency room during their follow up despite all of the risks and life threating character of their situation were told them. First of these patients were applied with back pain and syncope, the aortic diameter was measured between normal range and was followed by electrocardiogram and prediagnosis was the acute coronary syndrome. Three of the remaining 4 patients aortic dissection were detected by imaging ( 2 of them were DeBakey type 3 , one of them was DeBakey type 1). The last patient had AAA diagnosis with intraaortic thrombus.

\section{DISCUSSION}

AAA is a true surgical emergency which is needed to diagnose urgently and has high mortality when it's missed, its morbidity and mortality can be reduced with early intervention [15]. In a cohort study, which includes 73,450 patients and evaluates prevalence and risk factors of AAA, smoking incidence was found as $75 \%$. In our study smoking incidence was found as $70.2 \%$ at AAA patients and it is determined as an important factor. Hypertension is also evaluated as the independent risk factor for the rupture and dissection of AAAs [16]. Hypertension accompanied in the
$82.9 \%$ of 47 AAA patients in our study. This ratio is similar with other studies. In Edinburg study which investigates peripheral artery diseases, hypertension accompanied to AAA in the 34 patients of 1,592 [17]. In our study, known peripheral artery disease was recorded at two patients and after investigations, AAA was detected at these patients. Another risk factor is positive family history for AAA [18]. There was no family history in patients who included our study.

US is an imaging method which is commonly preferred at AAA diagnosis. It is a non-invasive, lowcost and accessible method so it has application area for diagnosis at emergency departments. US's primary benefit is abdominal aortic aneurysm can be investigated correctly, quickly and sensitively at the bedside [13].

US sensitivity for detecting AAA is found as $100 \%$ in many studies $[15,18,19]$. In obese people and at the presence of severe abdominal gas, inadequate imaging can be performed and US is dependent to the person who performs it.

In the study of Kuhn et al. [15]; AAA detecting sensitivity and specificity of US at patients who had abdominal pain and are more than 50 years old were found as $100 \%$, which is performed by emergency physicians who finished 3 days education program. In another study which was performed by Tayal et al. [20], US's positive predictive value was detected as $95 \%$ and the negative predictive value was detected as $100 \%$. In our study, ultrasound was performed by three years emergency medicine residents and they finished one-day US education program. US sensitivity was detected as $100 \%$ and specificity was detected as $91 \%$ at AAA detecting in the emergency department in our study.

In suspected cases, the aneurysm can be excluded if aortic diameter is evaluated as normal with US [21, 22]. In our study, patients who had aneurysmatic dilatation diagnosis with bedside ultrasound could be followed up by cardiovascular surgery service. Detecting operation needs according to annual growth rate provided a base for viewing mortality rates at follow-ups.

Nephrolithiasis took the first place at misdiagnoses [23]. In our study after the clinical suspect, we performed CT imaging to some patients and $10 \%$ of them had nephrolithiasis. The similarity of pain characteristic and not the occurrence of co- 
symptoms at the presence of risk factors cause the need of additional imaging methods for confirming the diagnosis. Co-diagnoses which were achieved by CT imaging which was performed for the suspect of AAA, was nephrolithiasis $(13 \%)$, cholelithiasis $(2.6 \%)$ and renal infarct $(1.3 \%)$ respectively.

Usage of US as a screen test for AAA diagnosis was considering in a close future [13]. Early surgical consultation and intervention can be possible in the presence of rupture or dissection with abdominal aortic evaluation by US. At the presence of uncomplicated aneurysms, US can be used for follow up and detecting the time of elective surgery.

Bedside US can be done simultaneously with resuscitation or after the proper treatment because it is non-invasive and easily applicable.

As a result, the most important role of the emergency physician is detecting if there is a need for emergent surgical intervention or medical treatment. Etiology can be detected in many of the patients with detailed anamnesis which includes all of the pain's characteristics, proper physical examination, laboratory and radiologic investigations which are requested with correct indications. At this stage, patient's present clinical situation must be taught for deciding usage of imaging methods and if the patient is unstable could not be taken from the emergency room.

\section{Limitations}

This study has some limitations which have to be pointed out. First the small patient population do not allow us to draw any conclusion about the effectiveness of this technique. Furthermore, the follow-up was limited. Larger series are needed to confirm the effectiveness of the bedside ultrasound imaging in detecting aortic aneurysms. Second we have high aortic aneurysm and dissection rates according to the literatüre this may be due to narrow range of age and symptomatology of selected patients.

\section{CONCLUSION}

If there is AAA in the suspected diagnosis list, evaluation of an aortic aneurysm with bedside US is necessary and diagnostic. Aortic imaging in patients enables detecting of AAA, dissection diagnoses and rupture presence. Evaluating aorta as normal indicates the necessity of considering additional pathologies in the differential diagnosis. Thus, investigation and time can be used properly and adequate treatment steps can be chosen for patients. Bedside ultrasonographic imaging must be routinized by emergency medicine physicians for using time and investigation numbers properly.

\section{Conflict of interest}

The authors disclosed no conflict of interest during the preparation or publication of this manuscript.

\section{Financing}

The authors disclosed that they did not receive any grant during conduction or writing of this study.

\section{REFERENCES}

[1] Graff LG 4th, Robinson D. Abdominal pain and emergency department evaluation. Emerg Med Clin North Am 2001;19:12336.

[2] Lameris W, Randen A, Dijkdraaf MG, Bossuyt PM, Stoker J, Boermeester MA. Optimization of diagnostic imaging use in patients with acute abdominal pain (OPTIMA): Design and rationale. BMC Emerg Med 2007;7:9.

[3] Majumper PP, St Jean PL, Ferrell RE, Webster MW, Steed DL. On the inheritance of abdominal aortic aneurysm. Am J Hum Genet 1991;48:164-70.

[4] Bickerstaff LK, Pailero PC, Hollier LH, Melton LJ, Van Peenen HJ, Cherry KJ, et al. Thoracic aortic aneurysms: a population based study. Surgery 1982;92:1103-8.

[5] Soysal M, Denizci U, Bayar R, Keşmer Y, Ulusoy M, Söylemez H, et al. Yaşlı popülasyonda abdominal aort anevrizmasısıklığının ve kardiyovasküler risk faktörleri ile ilişkisinin belirlenmesi. Klinik Gelişim 1996;9:4110-5.

[6] Coady A, Rizza JA, Hammond GL, Mandapati D, Darr U, Kopf GS, et al. What is appropriate size criterion for resection of thoracic aortic aneurysm? J Thorac Cardiovasc Surg 1997;113:476-91.

[7] Pasipoularides A, Murgo JP, Bird JJ, Craig WE. Fluid dynomics of aortic stenosis: mehanisms for the presence of subvalvular pressure gradients. Am J Physiol 1984;246(4 Pt 2):H542-50.

[8] Svensson LG Crawford ES (eds). Cardiovascular and vascular disease of the aorta. 1st edition. Philidelphia: WB Saunders Company; 1997.

[9] Strachan DP. Predictors of death from aortic aneurysm among middle-aged men: the White Hall Study. Br J Surg 1991;78:4014.

[10] MacSeveney ST, Ellis M, Worrell PC, Greenhalgh RM, Powell JT. Smoking and growth rate of small abdominal aortic 
aneurysms. Lancet 1994;344:651-2.

[11] Lobato AC, Puceh-Leao P. Predictive factors for rupture of thoracoabdominal aortic aneurysms. J Vasc Surg 1998;27:44653.

[12] DeSanctis RW, Doroghazi RM, Austen WG, Buckley MJ. Aortic dissection. N Engl J Med 1987;317:1060-67.

[13] Barkin AZ, Rosen CL. Ultrasound detection of abdominal aortic aneurysm. Emerg Med Clin North Am 2004;22:675-8.

[14] American College of Emergency Physicians. Emergency ultrasonography imaging criteria compendum. ACEP Policy Statements. Ann Emerg Med 2009;53:550-70.

[15] Kuhn M, Bonnin RLL, Davey MJ, Rowland JL, Langlois SL. Emergency department ultrasound scanning for abdominal aortic aneurysm: accessible, accurate and advantageous. Ann Emerg Med 2000;36:219-23.

[16] Rodin MB, Daviglus ML, Wong GC, Liu K, Garside DB, Greenland $\mathrm{P}$, et al. Middle age cardiovasculer risk faktors and abdominal aortic aneurysm in older age. Hypertension 2003;42:61-8.

[17] Fowkes FG, Housley E, Cawosa FH, Macintyre CC, Ruckley CV, Prescott RJ. Edinburg Artery Study: prevelance of asymptomatic and symptomatic peripheral arterial disease in the general population. Int J Epidemiol 1991;20:384-92.

[18] Vowden P, Wilkinson D, Ausobsky JR, Kester RC. A comparison of three imaging techniques in the assessment of an abdominal aortic aneurysm. J Cardiovasc Surg (Torino) 1989;30:891-6.

[19] Ernst CB. Abdominal aortic aneurysm. N Engl J Med 1993;328:1167-72.

[20] Tayal VS, Graf CD, Gibbs MA. Prospective study of accuracy and outcome of emergency ultrasound for abdominal aortic aneurysm over two years. Acad Emerg Med 2003;10:86771.

[21] Knaut AL, Kendall JL, Patten R, Ray C. Ultrasonographic measurement of aortic diameter by emergency physicians approximates results obtained by computed tomography. J Emerg Med 2005;28:119-26.

[22] Walker A, Brenchley J, Sloan JP, Lalanda M, Venables H. Ultrasound by emergency physicians to detect abdominal aortic aneurysms: a UK case series. Emerg Med J 2004;21:257-9.

[23] Rose WM III, Ernst CB. Abdominal aortic aneurysm. Comp Ther 1995;21:339-43. 